\title{
Underrepresentation of vulnerable older patients with cancer in phase II and III oncology registration trials: a case-control study
}

Laura Tack $^{\mathrm{a}}$, Tessa Lefebvre ${ }^{\mathrm{a}, \mathrm{b}}$, Michelle Lycke ${ }^{\mathrm{a}}$, Lies Pottel ${ }^{\mathrm{a}}$, Lieselot Cool ${ }^{\mathrm{a}}$, Lore Ketelaars ${ }^{\mathrm{c}}$, Jolien De Zutter $^{\mathrm{c}}$, Evi Martens ${ }^{\mathrm{c}}$, Hans Pottel ${ }^{\mathrm{d}}$, Karin Stellamans ${ }^{\mathrm{a}}$, Koen Van Eygen ${ }^{\mathrm{a}}$, Patrick Werbrouck ${ }^{\mathrm{e}}$, Philippe Vergauwe $^{\mathrm{f}}$, Hans Wildiers ${ }^{\mathrm{g}}$, Patricia Schofield ${ }^{\mathrm{h}, \mathrm{i}}$, Tom Boterberg ${ }^{\mathrm{b}}$, and Philip R. Debruyne ${ }^{\mathrm{a}, \mathrm{i}}$

\footnotetext{
${ }^{a}$ Department of Medical Oncology, Cancer Centre, General Hospital Groeninge, Kortrijk, Belgium

${ }^{\mathrm{b}}$ Department of Radiation Oncology and Experimental Cancer Research, Ghent University, Ghent, Belgium

${ }^{c}$ Department of Psychology, General Hospital Groeninge, Kortrijk, Belgium

${ }^{\mathrm{d}}$ Department of Public Health and Primary Care @ Kulak, Catholic University Leuven Kulak, Kortrijk, Belgium

${ }^{e}$ Department of Urology, General Hospital Groeninge, Kortrijk, Belgium

${ }^{\mathrm{f}}$ Department of Gastro-enterology, General Hospital Groeninge, Kortrijk, Belgium

${ }^{g}$ Department of Medical Oncology, University Hospital Leuven, Leuven, Belgium

${ }^{\mathrm{h}}$ Sheffield Hallam University, Sheffield, UK

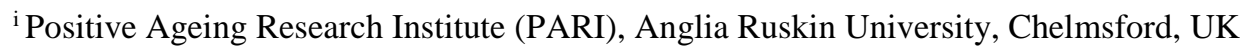

\section{Corresponding author:}

Prof. Philip Debruyne, Kortrijk Cancer Centre, General Hospital Groeninge, Pres. Kennedylaan 4, B-8500 Kortrijk, Belgium; E: Philip.Debruyne@azgroeninge.be; T: +32 (0)56 6339 00;

F: +32 (0)56 633909

\section{Conflict of interest:}

The authors have no conflict of interest to declare.

\section{Acknowledgements:}

Our work was supported by the Cancer Plan Actions of the Belgian Federal Government (NKP_24_018, NKP_CA_04, and NKP_2122c_044), and the Kortrijk Oncology Fund, King Baudouin Foundation, Brussels, Belgium. 


\begin{abstract}
Objectives

We aimed to determine the proportion of "fit" versus "vulnerable" older patients with cancer included in phase II and III oncology registration trials, as compared to the proportions in a real life oncology setting.
\end{abstract}

\title{
Methods
}

Trial and patient characteristics of older ( $\geq 70$ years) patients treated at the OECI-designated clinical cancer centre in Kortrijk and included in a phase II or III oncology registration trial were collected retrospectively. These patients were matched individually with randomly-selected patients from the general oncology setting, based on gender, age, tumour type, tumour stage, and treatment intent. Patients' fitness, based on routine Geriatric-8 (G8) screening, was retrieved from prospectively constructed databases.

\section{Results}

Between November 2012 and October 2018, 218 older patients with cancer were included in a phase II or III oncology registration trial. Of those, 41 cases with a mean age of 76,0 years were included in the analyses. A Fisher's Exact Test revealed a statistical significant difference between cases and matched controls, with a higher proportion of "fit" patients included in phase II or III oncology registration trials compared to the proportion in the matched control group (respectively $70.7 \%$ and $41.5 \%, \mathrm{p}<0.010$ ).

\section{Discussion}

We provide evidence for the hypothesis that older patients included in phase II or III oncology trials are significantly fitter than the real life oncology population. Some form of geriatric evaluation should be integrated in future cancer clinical trials to enable stratification according to this parameter and allow subgroup analysis. This will broaden the application and interpretation of trial results. 


\section{Introduction}

With the current demographic revolution, a result of the ageing of populations, epidemiologic data have predicted that the cancer incidence in older ( $\geq 65$ years) adults will increase with $67 \%$ by 2030 in the United States. ${ }^{1}$ Despite the continued increase in cancer incidence in older individuals, data from large cooperative groups have shown that only 22 to $32 \%$ of the older patient population participates in trials for cancer therapy. ${ }^{2-4}$ However, the majority of older adults enrolled on clinical trials have a good Eastern Cooperative Oncology Group Performance Status (ECOG PS) which makes them not representative for the majority of patients seen in the clinic. The oncogeriatric population is very heterogeneous, and based on their general health status they can be categorized as "fit", "vulnerable", or "frail", which respectively corresponds to functionally independent patients, those with increased risk of developing dependency, and those who have a minimal functional reserve. ${ }^{5-7}$

Phase II and III registration trials are designed for the approval of new drug applications (NDA) or supplemental new drug applications (sNDA) for the extension of indications and/or posology. As the inclusion and exclusion criteria are very strict, usually only fit older patients can be enrolled ${ }^{8}$, limiting the applicability of evidence-based recommendations within the field of oncology to older patients with cancer who may have a vulnerable or frail profile. ${ }^{9,10}$ In geriatric oncology, clinical judgement alone is not sufficient for optimal treatment planning. Before its initiation, oncologists must be aware of agerelated changes and identify the subset of patients who are vulnerable and at risk of increased treatment toxicity in order to ensure effective and safe cancer treatment in this patient population. ${ }^{11-13}$

The National Comprehensive Cancer Network (NCCN), the International Society of Geriatric Oncology (SIOG) and the American Society of Clinical Oncology (ASCO) recommend a geriatric evaluation of all patients with cancer aged 65 years and older. ${ }^{14-16}$ They suggest a two-step method consisting of a short screening tool to select patients who need further multidisciplinary evaluation to guide treatmentrelated decisions as well as to implement geriatric interventions. ${ }^{14,17-20}$ In the General Hospital Groeninge, we opt for the Geriatric-8 (G8) as a first step screening tool to identify older patients with cancer who would benefit from a more in-depth evaluation. The latter, also referred to as a comprehensive geriatric assessment (CGA), represents the second step. The G8 alone, however, appears to be predictive for functional decline and overall survival. ${ }^{21-23}$ Older adults with cancer, characterized as fit from this screening, are assumed to benefit from the same treatment as younger patients. If these patients could be separated from those that would not benefit or even have a detrimental effect of treatment, treatment side effects and survival loss could be decreased in the latter population. The CGA is a comprehensive measure to recognize heterogeneity among older adults and to characterize the "functional age" of an older patient, allowing individualized approaches for cancer treatment. Furthermore, it detects unsuspected conditions in more than $50 \%$ of patients older than 65 years that may affect their ability to complete cancer treatment. ${ }^{24-26}$

Over the past few years, Dr. Arti Hurria advocated for the accrual of (vulnerable) older patients with cancer in clinical trials to improve the evidence base for treatments in this growing population. ${ }^{27-30} \mathrm{Her}$ research topics related to older adults with cancer and the use of a geriatric assessment (GA) had a considerable influence on our research perspectives. The past years, many academic studies on the clinical relevance of a CGA were carried out by our research group. ${ }^{31-34}$

At present, no data have been published concerning the proportion of fit versus vulnerable/frail older patients with cancer included in a clinical trial in a general hospital setting, compared to the real life population of older patients with cancer. Therefore, our aim was to evaluate these proportions of G8negative and G8-positive older patients with cancer recruited in phase II and III oncology registration trials. 


\section{Methods}

\subsection{Study population and design}

Patients were recruited upon presentation at the Kortrijk Geriatric Oncology Clinic. The General Hospital Groeninge is a 1054-bed non-for-profit public-private partnership (PPP) teaching hospital. Eligible patients needed to be $\geq 70$ years at the time of trial inclusion, be diagnosed with a histologically confirmed solid tumour or haematological malignancy (any stage and any type of treatment), be included in a phase II or III oncology registration trial at the Organisation of European Cancer Institutes (OECI)designated clinical cancer centre of the General Hospital Groeninge, Kortrijk, Belgium, and have been evaluated with the G8 screening tool or full CGA. All geriatric data needed to be collected within six months from trial inclusion. Patients included in elderly-specific phase II or III oncology registration trials were excluded. The online Appendix A summarizes the phase II/III trials included in analysis and their characteristics.

Patients recruited in phase II or III oncology registration trials were named 'cases' and have been matched to older patients from the general oncology setting included in the oncogeriatric database from the General Hospital Groeninge, further referred to as 'matched controls'. Cases and controls were matched if they were equal on following five criteria: gender, age, tumour type, tumour stage, and treatment intent. The research associate (L.T.) was not informed about their G8 (and CGA) result. If there was no match available in the database that fulfilled the matching criteria, cases were excluded from analysis.

The hypothesis of this study was formulated prospectively. Ethical approval was obtained by the local ethics committee of the General Hospital Groeninge (AZGS2015023). Formerly, the ethics committee approved registration of demographic, oncology and geriatric parameters within the framework of the KPC_24_A_025 (AZGS2012057), PROACTIVE (AZGS2012061), and REGERCAN (AZGS2015081) trials. These data have been retrospectively analysed within the scope of this research and have been registered without written consent in the oncogeriatric database since G8 and/or CGA are routine practice at our hospital (online Appendix B: Care Model Geriatric Oncology General Hospital Groeninge). Between November 2012 and October 2018, a mean of $65.6 \%$ of oncogeriatric patients with diagnosis and treatment at our hospital have received a GA (minimum coverage of $39.4 \%$, maximum coverage of $73.3 \%$ ).

\subsection{Measures}

Three onco-psychologists (L.K., J.D.Z., E.M.) and two research associates (L.P., M.L.) were qualified to conduct the G8 and/or CGA and are called trained healthcare workers (THCWs). Since G8 screening data are available for all patients, we defined patients with a G8 score of more than 14 as G8-negative (G8-) or fit and those who scored 14 or less as G8-positive (G8+) or vulnerable. . $^{18,22,23,35}$

In case of a positive screening ( $\mathrm{G} 8 \leq 14)$, a full CGA was conducted. The CGA examines different agerelated domains including comorbidities (Charlson Comorbidity Index $\left.(\mathrm{CCI})^{36}\right)$, polypharmacy (number of drugs), functional status (Activities of Daily Living (ADL), instrumental Activities of Daily Living $(\mathrm{iADL})^{37,38}$ ), cognition (mini-mental status examination (MMSE) or Freund Clock Drawing Test $(\mathrm{CDT})^{39,40}$ ), nutrition (Mini Nutritional Assessment - Short Form ${ }^{41}$ ), emotional status (Geriatric Depression scale $-15^{42}$ ) and physical status (number of falls). When one reaches the cut-off value of a certain CGA domain, this patient is suggested to be 'vulnerable' on that specific domain. Two 
definitions of vulnerability based upon CGA result were used: vulnerability in one or more domains and vulnerability in two or more domains. ${ }^{35,43}$

\subsection{Statistical analysis}

Descriptive statistics were performed to present patient and trial characteristics. In order to assess the comparability of both cohorts, also demographic and clinical data were statistically analysed by using an independent sample t-test or Exact Pearson Chi-Square test. The proportions of G8- and G8+ patients were assessed by a $2 \times 2$ Contingency table to determine the association between the two variables condition (G8-/G8+) and group (case/matched control). A Fisher's Exact test was used to compare the proportions of G8+ patients in the group of cases, matched controls and older patients with cancer included in the oncogeriatric database at the General Hospital Groeninge. P-values below 0.05 were considered statistically significant. All statistical analyses were conducted using Microsoft Office Excel 2013 (Microsoft, Inc., Redmond, WA) and IBM SPSS v.24 (SPSS, Inc., Chicago, IL) software.

\section{Results}

\subsection{Study population characteristics}

From November 2012 till October 2018, 3,017 older patients with cancer were evaluated by a G8 screening and/or CGA in the Kortrijk Geriatric Oncology Clinic. Within the same period, 688 patients had been included in phase II or III oncology registration trials. Of those, 380 patients were excluded as they were not 70 years or older, and another 116 patients had a protocol-specific screen failure for the respective trials. Next, another 170 were excluded as the $\mathrm{G} 8$ was not assessed $(\mathrm{N}=122)$ or not within six months from trial inclusion $(\mathrm{N}=48)$, five were part of an elderly-specific trial, and for two patients no match was possible. The remaining 41 cases were eligible for analysis (Fig. 1). Of all 41 patients, 18 had a geriatric evaluation before trial inclusion (median time 22 days), 4 had an assessment on the moment of trial inclusion, and 19 had an evaluation after they were included in a phase II or III clinical trial (median time 89 days).
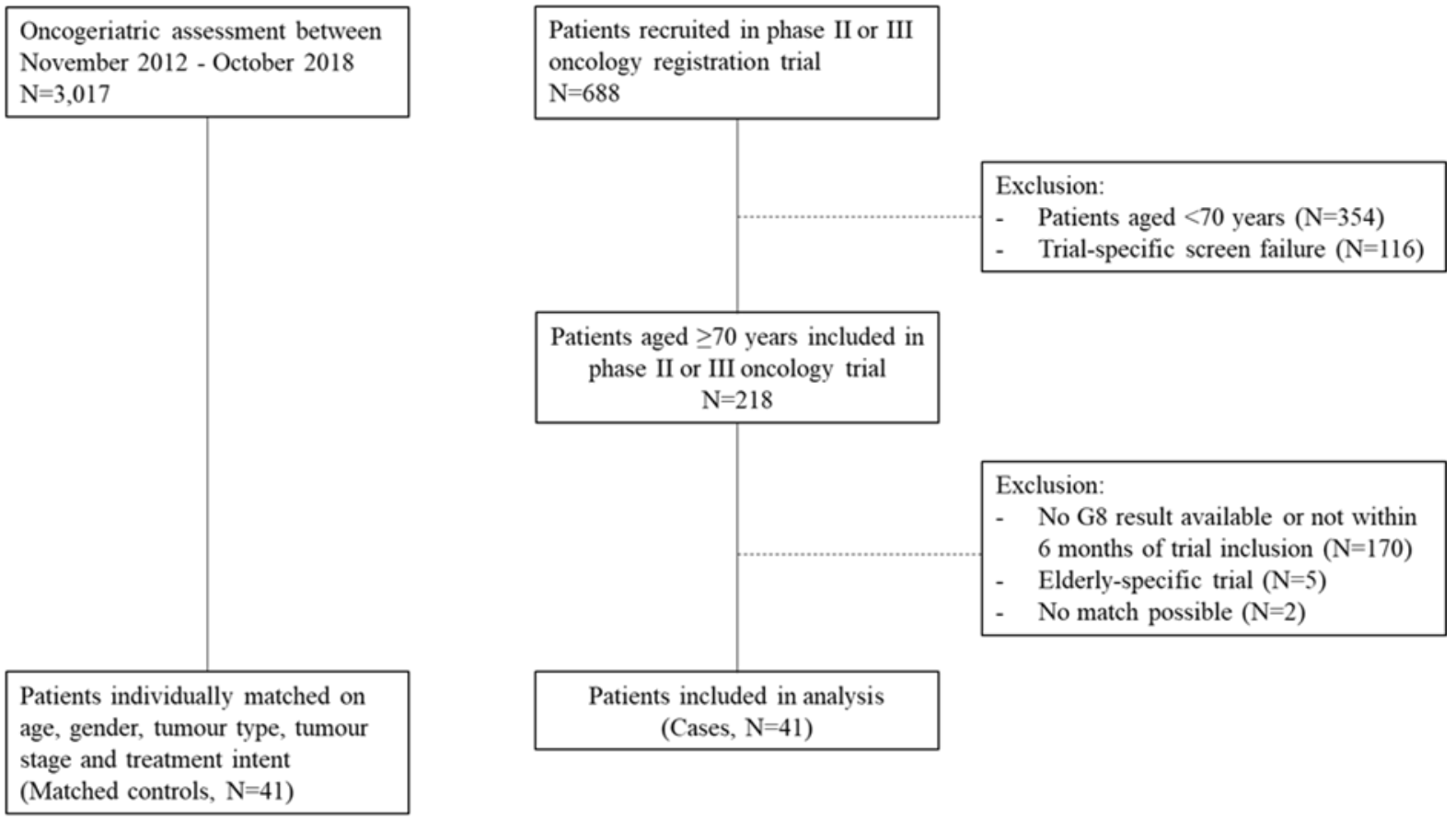

Fig. 1. Patient flow diagram. 
Cases had an average age of 76.0 years and matched controls had an average of 75.0 years (range for both cohorts: 70-86 years). The selected cohorts included more male than female individuals $(75.6 \%$ and $24.4 \%$, respectively). Within the case cohort, more patients were married in comparison to the control group ( $80.5 \%$ and $68.3 \%$, respectively), but the living situation was similar in both cohorts with $\geq 95.0 \%$ living at home. Professional homecare was used more by matched controls than by the cases (58.5\% and 39\%, respectively). Patients included in phase II or III oncology registration trials were diagnosed with the following malignant conditions: genitourinary (39.1\%), haematological malignancies (34.1\%), digestive tract (12.2\%), gynaecological (9.7\%), and breast (4.9\%). Most tumours were advanced $(51.2 \%)$ and a treatment with palliative intent was assigned to a majority of patients (58.5\%). Full demographic and oncology characteristics of the cases and matched controls are presented in Table 1. 
Table 1. Demographic and oncology characteristics of Cases and Matched controls

\begin{tabular}{|c|c|c|c|c|c|}
\hline & $\begin{array}{l}\text { No. of } \\
\text { Cases }\end{array}$ & $\%$ & $\begin{array}{c}\text { No. of } \\
\text { Matched controls }\end{array}$ & $\%$ & Significance \\
\hline \multicolumn{6}{|l|}{ Demographic characteristics } \\
\hline \multicolumn{6}{|l|}{ Sex } \\
\hline Male & 31 & 75.6 & 31 & 75.6 & \multirow{2}{*}{$p=1.00$} \\
\hline Female & 10 & 24.4 & 10 & 24.4 & \\
\hline \multicolumn{6}{|l|}{ Age, y } \\
\hline Mean (range) & \multicolumn{2}{|c|}{$76.0(70-86)$} & \multicolumn{2}{|c|}{$75.0(70-86)$} & $p=0.98$ \\
\hline \multicolumn{6}{|l|}{ Education: age, y } \\
\hline \multicolumn{6}{|l|}{ Marital Status } \\
\hline Married & 33 & 80.5 & 28 & 68.3 & $p=0.41 *$ \\
\hline Widow-er & 6 & 14.6 & 5 & 12.2 & \\
\hline Divorced & 1 & 2.4 & 3 & 7.3 & \\
\hline Single & 1 & 2.4 & 4 & 9.8 & \\
\hline Alone & 0 & 0.0 & 1 & 2.4 & \\
\hline \multicolumn{6}{|l|}{ Living situation } \\
\hline Home with partner & 32 & 78.0 & 27 & 65.9 & $p=0.71^{*}$ \\
\hline Home - alone & 7 & 17.1 & 10 & 24.4 & \\
\hline Home - with family member & 1 & 2.4 & 2 & 4.8 & \\
\hline Other & 1 & 2.4 & 2 & 4.8 & \\
\hline \multicolumn{6}{|l|}{ Professional homecare } \\
\hline Yes & 16 & 39.0 & 24 & 58.5 & $p=0.08$ \\
\hline No & 25 & 61.0 & 17 & 41.5 & \\
\hline \multicolumn{6}{|l|}{ Clinical characteristics } \\
\hline \multicolumn{6}{|l|}{ Primary tumour diagnosis } \\
\hline Breast & 2 & 4.9 & 2 & 4.9 & $p=1.00^{*}$ \\
\hline Digestive & 3 & 7.3 & 3 & 7.3 & \\
\hline Genitourinary - bladder & 4 & 9.8 & 4 & 9.8 & \\
\hline Genitourinary - prostate & 12 & 29.3 & 12 & 29.3 & \\
\hline Gynaecological - ovary and primary peritoneal carcinoma & 2 & 4.9 & 2 & 4.9 & \\
\hline Gynaecological - corpus uteri & 1 & 2.4 & 2 & 4.9 & \\
\hline Gynaecological - other & 1 & 2.4 & 0 & 0.0 & \\
\hline Haematological - non-hodgkin lymphoma & 11 & 26.8 & 11 & 26.8 & \\
\hline Haematological - chronic lymphocytic leukemia & 3 & 7.3 & 3 & 7.3 & \\
\hline \multicolumn{6}{|l|}{ Diagnosis } \\
\hline New diagnosis & 20 & 48.8 & 24 & 58.5 & $p=0.62 *$ \\
\hline Progression & 19 & 46.3 & 16 & 39.0 & \\
\hline Relapse & 2 & 4.9 & 1 & 2.4 & \\
\hline \multicolumn{6}{|l|}{ Tumour type } \\
\hline Early (I-II) & 6 & 14.6 & 6 & 14.6 & $p=1.00$ \\
\hline Advanced (III-IV) & 21 & 51.2 & 21 & 51.2 & \\
\hline Not reported & 14 & 34.1 & 14 & 34.1 & \\
\hline \multicolumn{6}{|l|}{ Treatment intent } \\
\hline Curative & 16 & 39.0 & 16 & 39.0 & $p=1.00^{*}$ \\
\hline Palliative & 24 & 58.5 & 24 & 58.5 & \\
\hline No active treatment/supportive care & 1 & 2.4 & 1 & 2.4 & \\
\hline
\end{tabular}

\subsection{Trial characteristics}

As illustrated in table 2, there were less phase II than phase III oncology registration trials included in this retrospective study (39.3\% and $60.7 \%$, respectively). Also, most of the trials were commercial (71.4\%). The clinical specialty groups of the trials included in our analysis were genitourinary tumours (35.7\%), haematological malignancies (32.1\%), digestive oncology (14.3\%), gynaecological malignancies $(7.1 \%)$, and breast cancer $(7.1 \%)$. Targeted therapy alone and in combination with 
chemotherapy were the most common types of investigational therapy ( $35.7 \%$ and $17.9 \%$, respectively). Targeted therapy included monoclonal antibodies (e.g. Rituximab), PARP inhibitors (e.g. niraparib), FGFR inhibitors (e.g. pemigatinib), tyrosine kinase inhibitors (e.g. ibrutinib), and CDK inhibitors (e.g. ribociclib). More information on the phase II/III oncology registration trials included in analysis can be found in the online Appendix A.

Table 2. Trial characteristics $(\mathrm{N}=28)$

\begin{tabular}{lll}
\hline & & $N(\%)$ \\
\hline Type of trial & Phase II & $11(39.3)$ \\
& Phase III & $17(60.7)$ \\
& Academic & $8(28.6)$ \\
& Commercial & $20(71.4)$ \\
& Breast & $2(7.1)$ \\
Clinical specialty group & Digestive & $4(14.3)$ \\
& Genitourinary - bladder & $2(7.1)$ \\
& Genitourinary - prostate & $8(28.6)$ \\
& Gynaecological & $2(7.1)$ \\
& Haematological oncology & $9(32.1)$ \\
Type of investigational therapy & Solid or haematological malignancies & $1(3.6)$ \\
Combination of drugs & & $5(17.9)$ \\
& Chemotherapy and molecular targeted therapy & $3(10.7)$ \\
Chemotherapy, radiotherapy, molecular targeted therapy & $1(3.6)$ \\
Chemotherapy & Chemotherapy, radiotherapy, hormonal therapy & $2(7.1)$ \\
Hother & Hormonal therapy and molecular targeted therapy & $3(10.7)$ \\
\hline
\end{tabular}

\subsection{Comparison of the proportion of vulnerable older patients in phase II or III oncology registration trials compared to the proportion in the general oncology setting}

As presented in figure 2, 29 cases and 17 matched control patients were categorized as G8- $(70.7 \%$ and $41.5 \%$, respectively) while 12 cases and 24 matched controls were judged as G8+ $(29.3 \%$ and $58.5 \%$, respectively). A contingency table was created to assess the association between the two variables, condition (G8-/G8+) and group (case/control). A two-tailed Fisher's Exact test indicated a significant difference between the proportions G8- and G8+ older patients with cancer in the case and matched control group ( $\mathrm{p}<0.05$ ), as well as between the proportions in the case group and oncogeriatric database $(\mathrm{p}<0.001)$.

The proportions of G8- and G8+ patients in the matched control group resembled the proportions of G8(43.9\%) and G8+ (56.1\%) older patients with cancer who had a geriatric screening between November 2012 and October 2018 and were included in the database $(\mathrm{p}=0.8744)$. 


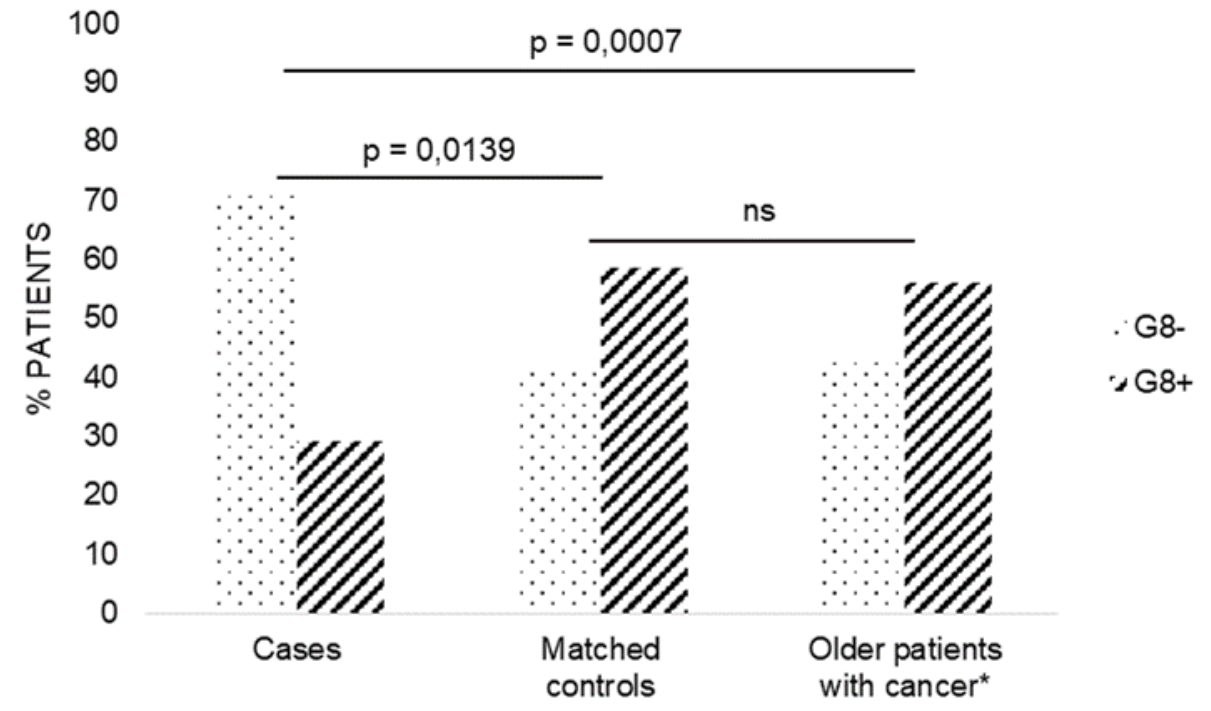

Fig. 2. Proportions of G8- and G8+ older patients with cancer in the group with cases, matched controls and patients included in the oncogeriatric database from the General Hospital Groeninge (*), determined by G8 score.

\subsection{Determination of the CGA domains in which geriatric vulnerabilities are most frequently detected}

Of all patients included, $29.3 \%$ of the cases (12/41) and 58.5\% of the matched controls (24/41) had a positive G8 screening. Consequently, THCWs performed a CGA. Although not all CGA data were complete, abnormal test results of the G8+ patients in the groups of cases, matched controls and older patients with cancer included in the oncogeriatric database were most frequently detected in the domains of community functioning $(58.3 \%, 75.0 \%$, and $84.5 \%$, respectively), nutrition $(58.3 \%, 65.2 \%$, and $72.6 \%$, respectively), and polypharmacy $(75.0 \%, 70.8 \%$, and $69.7 \%$, respectively). Based on the results of the CDT screening tool, G8+ cases showed a trend to score negative on the cognitive domain, in contrast to the control groups. However, all G8+ cases and matched controls scored negative on the MMSE (Table 3). 


\begin{tabular}{|c|c|c|c|c|c|c|c|c|c|}
\hline & Test & Range & Cut-off & $\begin{array}{l}\text { No. of } \\
\text { G8+ Cases } \\
\left(\mathrm{N}=12^{* *}\right)\end{array}$ & $\%$ & $\begin{array}{l}\text { No. of G8+ } \\
\text { Matched controls } \\
\quad\left(\mathrm{N}=24^{* *}\right)\end{array}$ & $\%$ & $\begin{array}{l}\text { No. of G8+ } \\
\text { older patients } \\
\text { with cancer* } \\
\left(\mathrm{N}=1693^{* *}\right)\end{array}$ & $\%$ \\
\hline $\begin{array}{l}\text { Functional status - } \\
\text { self care }\end{array}$ & $\mathrm{ADL}$ & $0-6$ & $\leq 5$ & $0 / 12$ & 0.0 & $0 / 24$ & 0.0 & $907 / 1668$ & 54.4 \\
\hline $\begin{array}{l}\text { Functional status - } \\
\text { community functioning }\end{array}$ & iADL & $0-8$ & $\leq 7$ & $7 / 12$ & 58.3 & $18 / 24$ & 75.0 & $1408 / 1666$ & 84.5 \\
\hline Physical status & No. of falls & NA & $\geq 1$ & $3 / 12$ & 25.0 & $5 / 23$ & 21.7 & $594 / 1662$ & 35.7 \\
\hline \multirow[t]{2}{*}{ Cognition } & MMSE & $0-30$ & $\leq 23$ & $0 / 6$ & 0.0 & $0 / 12$ & 0.0 & $239 / 734$ & 32.6 \\
\hline & CDT & $0-7$ & $\leq 4$ & $0 / 5$ & 0.0 & $5 / 13$ & 38.5 & $233 / 804$ & 29.0 \\
\hline Depression & GDS-15 & $0-15$ & $\geq 6$ & $2 / 10$ & 20.0 & $2 / 20$ & 10.0 & $230 / 1293$ & 17.8 \\
\hline Nutrition & MNA-SF & $0-14$ & $\leq 11$ & $7 / 12$ & 58.3 & $15 / 23$ & 65.2 & $1214 / 1673$ & 72.6 \\
\hline Co-morbidities & CCI & $0-37$ & $\geq 4$ & $2 / 12$ & 16.7 & $0 / 24$ & 0.0 & $453 / 1693$ & 26.8 \\
\hline Polypharmacy & $\begin{array}{l}\text { No. of } \\
\text { drugs }\end{array}$ & NA & $\geq 5$ & $9 / 12$ & 75.0 & $17 / 24$ & 70.8 & $1180 / 1692$ & 69.7 \\
\hline \multicolumn{10}{|c|}{$\begin{array}{l}\text { * patients included in the oncogeriatric database at the General Hospital Groeninge (November } 2012 \text { - October 2018) } \\
* * \text { number of vulnerable patients included in the cohort (not all CGA domains were completed) }\end{array}$} \\
\hline \multicolumn{10}{|c|}{$\begin{array}{l}\text { Abbreviations: ADL: Activities of Daily Living, iADL: instrumental Activities of Daily Living, MMSE: Mini Mental State Examination, CDT: } \\
\text { Clock Drawing Test, GDS-15: 15-item Geriatric Depression Scale, MNA-SF: Mini Nutritional Assessment-Short Form, CCI: Charlson Comorbidity } \\
\text { Index, NA: Not applicable. }\end{array}$} \\
\hline
\end{tabular}

The CGA conducted in G8+ patients enables the identification of vulnerable and frail profiles. In table 4, three categories within G8+ groups are displayed: vulnerable patients with a G8 score $\leq 14$, those with a G8 score $\leq 14$ and vulnerable in at least one domain of the CGA, and, last, patients with a G8 score $\leq 14$ and vulnerability in at least two CGA domains. A two-tailed Fisher's Exact test revealed a significant difference between cases and matched controls for first two categories $(p<0.05)$, but not for the last category $(\mathrm{p}=0.064)$. Still, the number of $\mathrm{G} 8+$ cases was significantly different from the older patients with cancer included in the oncogeriatric database for all three categories $(p<0.001)$. There was no significant difference observed between the matched controls and the patients included in the oncogeriatric database $(\mathrm{p}>0.05)$.

Table 4. Identification of vulnerability by G8 and CGA in Cases, Matched controls and the oncogeriatric population of the General Hospital Groeninge(*)

\begin{tabular}{llll}
\hline Patient characteristics & $\begin{array}{l}\text { Cases } \\
\mathrm{N}=41(\%)\end{array}$ & $\begin{array}{l}\text { Matched controls } \\
\mathrm{N}=41(\%)\end{array}$ & $\begin{array}{l}\text { Older patients with cancer* } \\
\mathrm{N}=3017(\%)\end{array}$ \\
\hline G8 $\leq 14$ & $12(29.3 \%)$ & $24(58.5 \%)$ & $1693(56.1)$ \\
G8 $\leq 14$ & $12(29.3 \%)$ & $24(58.5 \%)$ & $1685(55.9)$ \\
+1 CGA domain 'vulnerable' & $10(24.4 \%)$ & $19(46.3 \%)$ & $1563(51.8)$ \\
G8 $\leq 14$ & & \\
+2 CGA domains 'vulnerable' & & \\
$*$ patients included in the oncogeriatric database at the General Hospital Groeninge (November 2012 - October \\
$2018)$
\end{tabular}




\section{Discussion}

This paper aimed at identifying the proportion of G8+ older patients with cancer included in phase II/III oncology registration trials, compared to the proportion in a real life oncology setting. In this retrospective case-control study, at least $70 \%$ of older patients with cancer were designated fit by the G8 screening tool within six months from participation to a phase II or III oncology registration trial. This number is significantly different from the proportion of G8- older patients with cancer in the matched control group, as well as from the patients included in the oncogeriatric database. These data highlight the underrepresentation of vulnerable/frail older patients with cancer in phase II/III clinical trials.

The low recruitment of (vulnerable) older patients with cancer in clinical trials can be explained by strict eligibility criteria such as the ECOG PS and the exclusion of patients who are cognitively impaired..$^{2-}$ ${ }^{4,44,45}$ Studies have revealed that most older patients with cancer would forgo a potentially life-saving treatment if it impacted function or cognition. ${ }^{46,47}$ In our retrospective analysis, not all CGA data were complete and results on the cognitive domain should be interpreted cautiously as only half of the G8+ cases and G8+ matched controls fulfilled the CDT and MMSE. To allow a correct interpretation of table 3, absolute values of the number of G8+ cases, G8+ matched controls and G8+ older patients with cancer are indicated. Based on the results of the Freund CDT screening tool, the vulnerable patients included in phase II/III oncology registration trials seem to show a trend towards good cognitive functioning ${ }^{9,48}$, especially in comparison with the matched controls and patients included in the oncogeriatric database. When participating to a clinical trial, more support is mandatory in case of reduced cognitive functioning as it is essential one understands the trial and therapy side effects. Also, the majority of patients included in this study were treated with palliative intent. If these patients with an already vulnerable or frail profile would be forced into a curative treatment setting, they might suffer from symptoms not only caused by cancer, but also by treatment-induced toxicity. This points out the lack of evidence-based data related to the benefits and risks of cancer treatment in the vulnerable older patient as mainly older patients with a fit profile are enrolled in clinical oncology trials. ${ }^{49}$

Indeed, the evidence base concerning the effectiveness of treatment and potentially adverse consequences among older and/or frail adults remains sparse. Adaptation of trial designs to allow more vulnerable patients to receive upfront dose reductions or less intense regimens could be a useful intervention as treatment prescriptions for older adults with cancer are largely based on evidence generated in younger or fitter older adults. Therefore, Hurria et al. suggested an extension of patent exclusivity and post approval evaluation to administer effective and safe curative cancer treatment for older adults with cancer. ${ }^{50}$ In 2014, we introduced the concept of a dose-expansion cohort (DEC) dedicated in vulnerable older patients to be incorporated in phase $1 \mathrm{~b} / 2 \mathrm{a}$ protocols. ${ }^{51} \mathrm{~A}$ DEC offers the opportunity to reassess the efficacy, toxicity and maximally tolerated dose in these patients in the early phases of drug development. This modification of clinical trials for older patients was seconded by Dr. Arti Hurria, who was in favour of adding a cohort of older patients with cancer to the treatment arm that was shown to be superior, in order to evaluate the tolerability in older adults. ${ }^{28,52}$

Another adaptation of trials concerns the standard end-points such as disease-free survival or response rate (respectively in curative/adjuvant and palliative setting). For older adults with cancer, these are less applicable as they often die from other causes than the disease itself or from relapse. As daily functioning could offer a more accurate illustration of treatment outcome, it is suggested to integrate CGA parameters as surrogate trial end-points for older adults. Evaluation of geriatric parameters could contribute to better understanding the impact of new therapies on older individuals with cancer and to improving care in this vulnerable population. ${ }^{49,53}$ This case-control study advocates for the application 
of a GA before treatment start as it offers a correct estimation of an older person's treatment tolerance and possible benefit.

In this study, we investigated in which CGA domains the two separate cohorts of G8+ cases and matched controls most often reached the assigned cut-off values. Community functioning (iADL), nutrition and polypharmacy appeared as most sensitive CGA domains. This outcome acknowledges previous literature pointing out iADL and nutritional status as strong individual prognostic factors for overall survival in patients with cancer. ${ }^{18,54}$ Ideally, this outcome should be confirmed with a multicentre prospective cohort study.

To date, there is no consensus on a definition of frailty for oncology trials. Definitions in the general geriatric population are not specifically for older adults with cancer limiting their applicability. In this study, iADL, nutrition and polypharmacy were the CGA domains on which most G8+ patients reached the threshold, but it is plausible to consider other commonly applied criteria for the classification of patients as fit, vulnerable or frail. The EORTC Minimal Dataset interprets $\geq 1$ dependency in G8, iADL, CCI, and Social Situation as vulnerability ${ }^{20,49}$, while the NCCN older adult oncology guidelines judge elderly as vulnerable if they are ADL and/or iADL positive and as frail if they appear vulnerable on ADL, iADL, CCI, and/or physical, nutritional, affective, and cognitive domain. ${ }^{14}$ For Droz et al. (2010), significant comorbidity, iADL dependence, and severe malnutrition are the criteria to judge one vulnerable or frail, depending on the grade of comorbidity. ${ }^{55}$

According to Hamaker et al. (2012), an older patient with cancer is considered vulnerable when impairment is detected in at least two CGA domains ${ }^{35}$, while Soubeyran et al. (2011) used a less stringent definition as dependence in at least one domain is sufficient. ${ }^{43}$ As indicated in table 4, there is only a small difference between older patients who are G8+ only and those who are G8+ and vulnerable in one or two CGA domains, and our conclusions remain the same regardless of the definition used. The easiest way to define vulnerability is a G8 score of 14 or less. In our hospital, THCWs conduct a full CGA in G8+ patients, or on specific request of the physician. Regarding the clinical significance of a CGA, the appropriate judgement of THCWs is crucial to identify vulnerable or frail patients. The number of positive CGA domains to define frailty remains undetermined in geriatric oncology. For future research, we will keep track of which domains could be decisive in order to define frailty.

Some considerations need to be made when interpreting these results. First, this case-control study was monocentric and only patients included in phase II or III clinical trials that took place in the General Hospital Groeninge were selected. Second, the analysed sample size is relatively small due to the (strong) inclusion and exclusion criteria and the retrospective nature of this study. Although G8 screening and CGA are routine practice at our cancer centre, not all data were complete or gathered within six months from trial inclusion. Additionally, we were unable to match two patients according to the five matching criteria (age, gender, tumour type, tumour stage and treatment intent). Third, the selection of phase II/III trials might not be a convenient representation for the oncology registration trials in a real life oncology setting. For example, breast cancer is far more frequent than gynaecological tumours, but in this case-control study, results showed the opposite. This can be explained by trials' availability to include patients and, again, the strict inclusion criterion of a G8 result available within six months from trial inclusion. At last, $75 \%$ of patients included in analysis were male. This underrepresentation of women in our analysis can be largely explained by the imbalance in trials. When six patients were confronted with breast or gynaecological malignant condition, there were twelve treated for prostate cancer, which does not correspond with demographic characteristics. Therefore, case-control matching was performed. 
Some controls were conducted to confirm the generalizability of our results. First, the recent G8 result enables a correct estimation of trial patients' fitness which is decisive for the interpretation of study results. More importantly, based on five crucial parameters, cases have been matched to control patients included in the oncogeriatric database. This case-control matching corrects for any trial under- or overrepresentation and ensures a correct representation of the real life oncology population. Moreover, the proportions of G8- and G8+ older patients included in the matched control group resemble closely the proportions of older patients with cancer included in the oncogeriatric database, which suggests that the matched controls are representative for the older patients with cancer in a real life oncology setting. Furthermore, demographic and clinical characteristics of the excluded patients with CGA result available $(\mathrm{N}=48)$ have been analysed. An independent sample t-test and Exact Pearson Chi-Square test showed there was no statistically significant difference between the excluded patients and the cases, neither for the matching criteria gender $(\mathrm{p}=0.5)$, age $(\mathrm{p}=0.6)$, tumour type $(\mathrm{p}=0.1)$, tumour stage $(\mathrm{p}=0.5)$, and treatment intent $(\mathrm{p}=0.8)$, nor for the number of $\mathrm{G} 8+$ versus $\mathrm{G} 8$ - cases $(\mathrm{p}=0.2)$ (data not shown). The majority of the excluded patients $(56.1 \%)$ had a fit profile. However, this G8 result was obtained more than 6 months before or after trial inclusion, therefore no conclusions can be drawn.

To the best of our knowledge, we are the first to publish quantitative data on the proportion of vulnerable older patients with cancer included in clinical trials, in comparison to the real life population. We believe our data support the inclusion of G8 and/or CGA for oncogeriatric patients enrolled in clinical trials to improve adequate patient selection, risk stratification, and efficacy and safety evaluation. Agreement on a (more or less) uniform and straightforward evaluation of the fitness of the older population such as the G8 could contribute to better characterized patient populations, avoiding selection bias caused by the heterogeneity of the population.

In conclusion, this case-control study provides evidence for the hypothesis that older patients included in phase II or III oncology trials are significantly fitter than the real life oncology population. Integration of the G8 screening tool and/or CGA in future cancer clinical trials could enable stratification according to this parameter and allow subgroup analysis. New approaches to tackle the underrepresentation of vulnerable older patients with cancer in clinical trials are needed such as DECs in early phase trials and extra cohorts in later phase trials. This will broaden the application and interpretation of trial results.

\section{Conflict of interest}

The authors have no conflict of interest to declare.

\section{Contributions}

All authors have contributed substantially to the conception and design, acquisition of data and/or the analyses and interpretation of data, and manuscript writing. All authors have reviewed and approved this manuscript. 


\section{References}

1. Smith BD, Smith GL, Hurria A, Hortobagyi GN, Buchholz TA. Future of cancer incidence in the United States: burdens upon an aging, changing nation. J Clin Oncol. 2009;27(17):27582765.

2. Hutchins LF, Unger JM, Crowley JJ, Coltman CA, Jr., Albain KS. Underrepresentation of patients 65 years of age or older in cancer-treatment trials. N Engl J Med. 1999;341(27):20612067.

3. Yee KW, Pater JL, Pho L, Zee B, Siu LL. Enrollment of older patients in cancer treatment trials in Canada: why is age a barrier? J Clin Oncol. 2003;21(8):1618-1623.

4. Lewis JH, Kilgore ML, Goldman DP, et al. Participation of patients 65 years of age or older in cancer clinical trials. J Clin Oncol. 2003;21(7):1383-1389.

5. Balducci L, Stanta G. Cancer in the frail patient. A coming epidemic. Hematol Oncol Clin North Am. 2000;14(1):235-250, xi.

6. Balducci L, Extermann M. Management of cancer in the older person: a practical approach. Oncologist. 2000;5(3):224-237.

7. Serraino D, Fratino L, Zagonel V. Prevalence of functional disability among elderly patients with cancer. Crit Rev Oncol Hematol. 2001;39(3):269-273.

8. Brunello A, Sandri R, Extermann M. Multidimensional geriatric evaluation for older cancer patients as a clinical and research tool. Cancer Treat Rev. 2009;35(6):487-492.

9. Ketelaars L, Pottel L, Lycke M, et al. Use of the Freund clock drawing test within the mini$\operatorname{cog}$ as a screening tool for cognitive impairment in elderly patients with or without cancer. $J$ Geriatr Oncol. 2013;4:174-182.

10. Pottel L, Lycke M, Boterberg T, et al. Serial comprehensive geriatric assessment in elderly head and neck cancer patients undergoing curative radiotherapy identifies evolution of multidimensional health problems and is indicative of quality of life. Eur J Cancer Care (Engl). 2014;23(3):401-412.

11. Mohile SG, Bylow K, Dale W, et al. A pilot study of the vulnerable elders survey-13 compared with the comprehensive geriatric assessment for identifying disability in older patients with prostate cancer who receive androgen ablation. Cancer. 2007;109(4):802-810.

12. Terret C, Zulian GB, Naiem A, Albrand G. Multidisciplinary approach to the geriatric oncology patient. J Clin Oncol. 2007;25(14):1876-1881.

13. Terret C. How and why to perform a geriatric assessment in clinical practice. Ann Oncol. 2008;19 Suppl 7(7):vii300-303.

14. Network NCC. NCCN Clinical Practice Guidelines in Oncology: Older Adult Oncology. https://www.nccn.org/professionals/physician gls/pdf/senior.pdf. Published 2019. Accessed.

15. Wildiers H, Heeren P, Puts M, et al. International Society of Geriatric Oncology consensus on geriatric assessment in older patients with cancer. J Clin Oncol. 2014;32(24):2595-2603.

16. Mohile SG, Dale W, Somerfield MR, et al. Practical Assessment and Management of Vulnerabilities in Older Patients Receiving Chemotherapy: ASCO Guideline for Geriatric Oncology. J Clin Oncol. 2018;36(22):2326-2347.

17. Hurria A, Wildes T, Blair SL, et al. Senior adult oncology, version 2.2014: clinical practice guidelines in oncology. J Natl Compr Canc Netw. 2014;12(1):82-126.

18. Kenis C, Decoster L, Van Puyvelde K, et al. Performance of two geriatric screening tools in older patients with cancer. J Clin Oncol. 2014;32(1):19-26.

19. Extermann M, Aapro M, Bernabei R, et al. Use of comprehensive geriatric assessment in older cancer patients: recommendations from the task force on CGA of the International Society of Geriatric Oncology (SIOG). Crit Rev Oncol Hematol. 2005;55(3):241-252.

20. Pallis AG, Fortpied C, Wedding U, et al. EORTC elderly task force position paper: approach to the older cancer patient. Eur J Cancer. 2010;46(9):1502-1513.

21. Chakiba C, Bellera C, Etchepare F, Mathoulin-Pelissier S, Rainfray M, Soubeyran P. The prognostic value of G8 for functional decline. J Geriatr Oncol. 2019.

22. Soubeyran P, Bellera C, Goyard J, et al. Screening for vulnerability in older cancer patients: the ONCODAGE Prospective Multicenter Cohort Study. PLoS One. 2014;9(12):e115060. 
23. Bellera CA, Rainfray M, Mathoulin-Pelissier S, et al. Screening older cancer patients: first evaluation of the G-8 geriatric screening tool. Ann Oncol. 2012;23(8):2166-2172.

24. Repetto L, Fratino L, Audisio RA, et al. Comprehensive geriatric assessment adds information to Eastern Cooperative Oncology Group performance status in elderly cancer patients: an Italian Group for Geriatric Oncology Study. J Clin Oncol. 2002;20(2):494-502.

25. Extermann M. Comprehensive geriatric assessment basics for the cancer professional. $J$ Oncol Manag. 2003;12(2):13-17.

26. Hurria A, Gupta S, Zauderer M, et al. Developing a cancer-specific geriatric assessment: a feasibility study. Cancer. 2005;104(9):1998-2005.

27. Freedman RA, Dockter TJ, Lafky JM, et al. Promoting Accrual of Older Patients with Cancer to Clinical Trials: An Alliance for Clinical Trials in Oncology Member Survey (A171602). Oncologist. 2018;23(9):1016-1023.

28. Hurria A, Dale W, Mooney M, et al. Designing therapeutic clinical trials for older and frail adults with cancer: U13 conference recommendations. J Clin Oncol. 2014;32(24):2587-2594.

29. Hurria A, Levit LA, Dale W, et al. Improving the Evidence Base for Treating Older Adults With Cancer: American Society of Clinical Oncology Statement. J Clin Oncol. 2015;33(32):3826-3833.

30. Scher KS, Hurria A. Under-representation of older adults in cancer registration trials: known problem, little progress. J Clin Oncol. 2012;30(17):2036-2038.

31. Lycke M, Martens E, Ketelaars L, et al. Detection of alcohol abuse in older patients with cancer: The integration of alcohol screening questionnaires in the comprehensive geriatric assessment. J Geriatr Oncol. 2019.

32. Lycke M, Ketelaars L, Martens E, et al. The added value of an assessment of the patient's hand grip strength to the comprehensive geriatric assessment in G8-abnormal older patients with cancer in routine practice. J Geriatr Oncol. 2019.

33. Lycke M, Debruyne PR, Lefebvre T, et al. The use of uHear to screen for hearing loss in older patients with cancer as part of a comprehensive geriatric assessment. Acta Clin Belg. 2018;73(2):132-138.

34. Pottel L, Lycke M, Boterberg T, et al. G-8 indicates overall and quality-adjusted survival in older head and neck cancer patients treated with curative radiochemotherapy. BMC Cancer. 2015; $15: 875$.

35. Hamaker ME, Jonker JM, de Rooij SE, Vos AG, Smorenburg CH, van Munster BC. Frailty screening methods for predicting outcome of a comprehensive geriatric assessment in elderly patients with cancer: a systematic review. Lancet Oncol. 2012;13(10):e437-444.

36. Charlson M, Szatrowski TP, Peterson J, Gold J. Validation of a combined comorbidity index. J Clin Epidemiol. 1994;47(11):1245-1251.

37. Katz S, Ford AB, Moskowitz RW, Jackson BA, Jaffe MW. Studies of Illness in the Aged. The Index of Adl: A Standardized Measure of Biological and Psychosocial Function. Jama. 1963;185:914-919.

38. Lawton MP, Brody EM. Assessment of older people: self-maintaining and instrumental activities of daily living. Gerontologist. 1969;9(3):179-186.

39. Folstein MF, Folstein SE, McHugh PR. "Mini-mental state". A practical method for grading the cognitive state of patients for the clinician. J Psychiatr Res. 1975;12(3):189-198.

40. Freund B, Gravenstein S, Ferris R, Burke BL, Shaheen E. Drawing clocks and driving cars. $J$ Gen Intern Med. 2005;20(3):240-244.

41. Rubenstein LZ, Harker JO, Salva A, Guigoz Y, Vellas B. Screening for undernutrition in geriatric practice: developing the short-form mini-nutritional assessment (MNA-SF). $J$ Gerontol A Biol Sci Med Sci. 2001;56(6):M366-372.

42. Yesavage JA. Geriatric Depression Scale. Psychopharmacol Bull. 1988;24(4):709-711.

43. Soubeyran P, Bellera C, Goyard J, et al. Validation of the G8 screening tool in geriatric oncology: the ONCODAGE project. Journal of Clinical Oncology. 2011;29(suppl; abstr 9001; 2011 ASCO Annual Meeting).

44. Kazmierska J. Do we protect or discriminate? Representation of senior adults in clinical trials. Reports of practical oncology and radiotherapy : journal of Greatpoland Cancer Center in Poznan and Polish Society of Radiation Oncology. 2012;18(1):6-10. 
45. Aapro MS, Kohne CH, Cohen HJ, Extermann M. Never too old? Age should not be a barrier to enrollment in cancer clinical trials. Oncologist. 2005;10(3):198-204.

46. Fried TR, Bradley EH, Towle VR, Allore H. Understanding the treatment preferences of seriously ill patients. N Engl J Med. 2002;346(14):1061-1066.

47. Soto Perez De Celis E, Li D, Sun C-L, et al. Patient-defined goals and preferences among older adults with cancer starting chemotherapy (CT). Journal of Clinical Oncology. 2018;36(15_suppl):10009-10009.

48. Lycke M, Ketelaars L, Boterberg T, et al. Validation of the Freund Clock Drawing Test as a screening tool to detect cognitive dysfunction in elderly cancer patients undergoing Comprehensive Geriatric Assessment. Psycho Oncology. 2014;23(10):1172-1177.

49. Wildiers H, Mauer M, Pallis A, et al. End points and trial design in geriatric oncology research: a joint European organisation for research and treatment of cancer--Alliance for Clinical Trials in Oncology--International Society Of Geriatric Oncology position article. Journal of clinical oncology : official journal of the American Society of Clinical Oncology. 2013;31(29):3711-3718.

50. Cohen HJ, Naylor M, Hurria A. Underrepresentation of older adults in cancer trials--reply. In: Jama. Vol 311. United States2014:966-967.

51. Debruyne PR, Knott VE, Weller D. Themes for our journal: 2014-2016. Eur J Cancer Care (Engl). 2014;23(3):285-287.

52. Hurria A. Dosing drugs in elderly patients with cancer. Clin Adv Hematol Oncol. 2018;16(9):606-608.

53. Hurria A, Mohile SG, Dale W. Research priorities in geriatric oncology: addressing the needs of an aging population. J Natl Compr Canc Netw. 2012;10(2):286-288.

54. Kenis C, Baitar A, Decoster L, et al. The added value of geriatric screening and assessment for predicting overall survival in older patients with cancer. Cancer. 2018;124(18):3753-3763.

55. Droz JP, Balducci L, Bolla M, et al. Management of prostate cancer in older men: recommendations of a working group of the International Society of Geriatric Oncology. BJU Int. 2010;106(4):462-469. 